\title{
Preoperative computed tomography-assessed skeletal muscle index is a novel prognostic factor in patients with hepatocellular carcinoma following hepatectomy: a meta-analysis
}

\author{
Liqian Xu ${ }^{1,2 \#}$, Yuxia Jing ${ }^{3 \#}$, Chen Zhao ${ }^{4}$, Qin Zhang ${ }^{1,2}$, Xiaohong Zhao ${ }^{1,2}$, Ji Yang ${ }^{1,2}$, Lizhen Wu ${ }^{1,2}$, \\ Yunmei Yang ${ }^{1,2}$
}

${ }^{1}$ Zhejiang Provincial Key Laboratory for Diagnosis and Treatment of Aging and Physic-chemical Injury Diseases, the First Affiliated Hospital, School of Medicine, Zhejiang University, Hangzhou, China; ${ }^{2}$ Department of Geriatrics, the First Affiliated Hospital, School of Medicine, Zhejiang University, Hangzhou, China; ${ }^{3}$ Department of Geriatrics, Tianjin Fifth Central Hospital, Tianjin, China; ${ }^{4}$ Institute of Physical Education, Huzhou University, Huzhou, China

Contributions: (I) Conception and design: L Xu, Q Zhang, Y Yang; (II) Administrative support: Y Yang, Q Zhang; (III) Provision of study materials or patients: J Yang, X Zhao, L Wu; (IV) Collection and assembly of data: Y Jing, C Zhao; (V) Data analysis and interpretation: L Xu, C Zhao; (VI) Manuscript writing: All authors; (VII) Final approval of manuscript: All authors.

"These authors contributed equally to this work.

Correspondence to: Yunmei Yang. Zhejiang Provincial Key Laboratory for Diagnosis and Treatment of Aging and Physic-chemical Injury Diseases, the First Affiliated Hospital, School of Medicine, Zhejiang University, Hangzhou, China; Department of Geriatrics, the First Affiliated Hospital, School of Medicine, Zhejiang University, Hangzhou, China. Email: 1194070@zju.edu.cn.

Background: Recently, several studies have examined the association between preoperative sarcopenia and prognosis evaluation in patients with hepatocellular carcinoma (HCC) undergoing hepatectomy. However, their conclusions remain ambiguous and controversial. Thus, we conducted a meta-analysis to assess the prognostic role of preoperative sarcopenia in patients with HCC undergoing hepatectomy.

Methods: We searched the existing literature reporting on the prognostic value of preoperative computed tomography (CT)-assessed sarcopenia for the survival of patients with HCC undergoing hepatectomy. The pooled hazard ratios (HRs) and corresponding 95\% confidence intervals (CIs) of overall survival (OS) and disease-free survival (DFS) were used to assess the prognostic value of preoperative sarcopenia in HCC patients. The associations between preoperative sarcopenia and clinicopathological characteristics were also evaluated.

Results: A total of six studies with 1,420 patients (including 458 sarcopenia and 962 non-sarcopenia patients) were included in the meta-analysis. The results showed that preoperative sarcopenia was significantly associated with poor OS ( $\mathrm{HR}=1.572,95 \% \mathrm{CI}: 1.342-1.840, \mathrm{P}=0)$ and shorter DFS $(\mathrm{HR}=1.544$, 95\% CI: 1.178-2.024, $\mathrm{P}=0.002)$ in patients with HCC undergoing hepatectomy. Preoperative sarcopenia was also significantly related to larger diameter tumors (WMD =0.598, 95\% CI: 0.216-0.980, P=0.002). The results of the sensitivity analysis were stable in this meta-analysis. Egger's tests revealed that there was no significant publication bias.

Conclusions: Sarcopenia appears to have significant adverse impacts on postoperative outcomes in patients with hepatocellular carcinoma following hepatectomy. However, further large-scale prospective studies are needed to confirm our findings.

Keywords: Preoperative sarcopenia; hepatocellular carcinoma (HCC); skeletal muscle mass index (SMI); overall survival (OS); disease-free survival (DFS)

Submitted Feb 27, 2020. Accepted for publication Jul 31, 2020.

doi: 10.21037/jgo-20-122

View this article at: http://dx.doi.org/10.21037/jgo-20-122 


\section{Introduction}

Hepatocellular carcinoma (HCC) is one of the most common malignant carcinomas and is also the fifth cause of cancer-related death worldwide (1). Although liver transplantation is a possible treatment for early-stage cases, the prohibitive costs and limited donors are prohibitive. Hepatectomy, radiofrequency ablation, and transarterial chemoembolization are more effective alternative treatment options to improve the survival rate of patients $(2,3)$. The clinical outcome of HCC is related to various factors, including liver function, tumor size, differentiation, tumor, node, metastasis (TNM) stage, and comorbidities $(4,5)$. Improving our understanding of perioperative management can reduce morbidity and mortality and improve overall survival (OS) and disease-free survival (DFS) for patients with HCC.

Sarcopenia is an age-related decline in muscle mass and strength and is a significant cause of the functional decline. It is related to chronic diseases such as osteoporosis, cirrhosis, and heart failure, as well as a variety of cancers, including HCC (6-14). As a preoperative risk assessment index, it has attracted extensive attention in recent years. So far, a large number of studies have explored the relationship between preoperative sarcopenia and postoperative prognosis in patients undergoing surgery for HCC (15-23). However, the previously reported results remain controversial and ambiguous. In 2013, Harimoto et al. (22) pointed out that sarcopenia was a risk factor for overall survival and recurrence-free survival in Japanese patients undergoing liver resection for HCC. Similar results have been reported in other studies $(20,23)$. However, in 2019, Kroh et al. (17) reported that preoperative sarcopenia was not a risk factor for poor postoperative survival in HCC patients. We conducted the present meta-analysis in order to accurately and comprehensively understand the relationship between preoperative skeletal muscle depletion and the prognosis of patients with surgically treated HCC.

We present the following article in accordance with the PRISMA reporting checklist (available at http://dx.doi. org/10.21037/jgo-20-122).

\section{Methods}

\section{Literature search strategy}

Literature searches were independently conducted by two authors (Yuxia Jing and Ji Yang), covering all articles published in PubMed, Embase, and Web of Science before
July 2020. The keywords were as follows: ("HCC" or "liver cancer" or "hepatocellular carcinoma" or "hepatoma" or "liver cell carcinoma"), ("sarcopenia" or "sarcopenic" or "skeletal muscle depletion" or "muscle index" or "muscle mass"), and ("hepatectomy" or "open liver resection" or "open hepatectomy" or "laparoscopy" or "resection" or "surgery" or "surgical"). Search results were limited to articles published in English. The reference list of retrieved articles was manually searched to obtain other potentially related publications. We evaluated all relevant publications to determine the most eligible literature. Disagreements between the two authors were resolved by a third reviewer (Liqian $\mathrm{Xu}$ ).

\section{Inclusion and exclusion criteria}

The following inclusion criteria were applied to the selection of articles for further meta-analysis: (I) studies exploring the relationship between preoperative sarcopenia and the survival of patients with HCC undergoing hepatectomy; (II) studies including at least one of the following outcomes: duration of surgery (in minutes), estimated blood loss (in milliliters), length of hospital stay (in days), overall postoperative complications, severe (Clavien-Dindo $\geq 3$ ) complications, and hazard ratios (HRs) of OS and/or DFS; (III) patients were examined regularly and followed up for at least 12 months; and (IV) if primary studies involved the same patients, the most recent or completed study was included. The exclusion criteria were as follows: (I) patients with other pathological types of liver cancer or metastatic liver cancer; (II) patients with postoperative sarcopenia or those not surgically treated; and (III) conference abstracts, review articles, case reports, and experiments.

\section{Data extraction}

The extracted data included: the author's last name, year of publication, country of the study population, study period, number and type of patients, study design, clinicopathological information (i.e., sex, age, body mass index (BMI), liver histology, Child-Pugh classification, tumor differentiation, tumor size, and TNM stage), preoperative parameters (i.e., skeletal muscle measured indexes and cut-off values for sarcopenia), intraoperative parameters (i.e., operation time and estimated blood loss), and postoperative parameters (i.e., overall complications, severe postoperative complications, time of stay in the hospital, and HRs of OS and DFS). If the HRs were not 
reported directly, they were calculated according to survival data or survival curves in primary articles using methods suggested by Parmar (24) and Tierney et al. (25). If both were available, survival data were preferred as errors could occur when extracting the data from the survival curve of the original article. Also, multivariate analyses were preferable when HRs appeared in both univariate and multivariate analyses, as multivariate analyses considered the possible confusion of exposure variables.

\section{Quality assessment of selected studies}

The Newcastle-Ottawa Scale (NOS) (Wells GA, Shea B, O'Connell D, Peterson J, Welch V, Losos M, et al.) was used to assess the quality of studies selected in our meta-analysis (available from http://www.ohri.ca/programs/clinical_ epidemiology/oxford.htm). Study quality assessment was conducted by two authors (Qin Zhang and Xiaohong Zhao), and mainly included the selection of cases, comparability of the cohort, and outcome assessment of exposure to risks. A score of 0-9 (or a star rating) was assigned to each study, and a study with score $\geq 6$ was considered to be of high quality. Disagreements between the two reviewers (above) were resolved by a third reviewer (Liqian $\mathrm{Xu}$ ).

\section{Statistical analysis}

Dichotomous data were analyzed using odds ratios (ORs). Continuous data measured on the same unit of measurement was expressed as the mean value and standard deviation and was analyzed using the weighted mean difference (WMD). HRs were used to analyze postoperative OS and DFS. All results were reported with $95 \%$ confidence intervals (CIs). The I-squared $\left(\mathrm{I}^{2}\right)$ test was used to evaluate the heterogeneity of the studies, and a fixed or random effects model was chosen on this basis. According to the Cochrane review guidelines, severe heterogeneity is signified by an $\mathrm{I}^{2}$ result of $>50 \%$, and in these cases, a random effects model was selected; otherwise, a fixed-effect model was employed. Subgroup analysis was implemented to explore the sources of heterogeneity, and sensitivity analysis was performed to evaluate the quality and consistency of the results by successively eliminating each included study. Publication bias was assessed using Egger's test and Begg's test. All data in this paper was processed by using the STATA 16.0 package (Stata Corporation, College Station, TX, USA). If $\mathrm{P}<0.05$, the results were considered statistically significant.

This study was a systematic review and meta-analysis of all published literature and institutional review deemed this study exempt from ethical approval.

\section{Results}

\section{Study selection}

Figure 1 shows the research selection process. A total of 922 potentially relevant articles were identified from database searches for further detailed evaluation. After browsing the titles and abstracts of these articles, 26 articles remained and were assessed further. Three of these studies were excluded because they included patients diagnosed with bile duct cancers; seven studies were excluded due to overlapping data; another four studies were excluded because they included patients who were treated with chemoradiotherapy instead of surgery. Also, some studies were excluded because no relevant data were extracted for analysis. Three studies were excluded because the patients were not grouped by sarcopenia; one was excluded because it investigated the presence of postoperative sarcopenia in patients; one was excluded because of a minimum follow-up duration $<12$ months; another one was excluded because there was no survival data. Finally, six studies $(17,18,21-23,26)$ with a total of 1,420 participants (458 with sarcopenia and 962 without sarcopenia) were included in the meta-analysis. In Hamaguchi et al. (26), there are two sets of data for OS and DFS in preoperative sarcopenia patients classified according to intramuscular adipose tissue content (IMAC) and skeletal muscle mass index (SMI).

\section{Study and patient characteristics}

Table 1 shows the baseline clinical and laboratory characteristics of all studies included in the meta-analysis, and Table 2 shows the postoperative clinical characteristics of all studies included in the meta-analysis. All the included studies were retrospective cohort studies. Most (all but one) of them were published in the last 5 years (22). The majority of the studies were conducted in Japan, with two studies carried out in Germany and France. All patients were outpatients. We marked the literature with the first author's name and the year of publication.

\section{Definition of sarcopenia}

Table 3 depicts the skeletal muscle measurement details on computed tomography (CT) and the cut-off values for sarcopenia in each study. All of the studies used CT imaging 


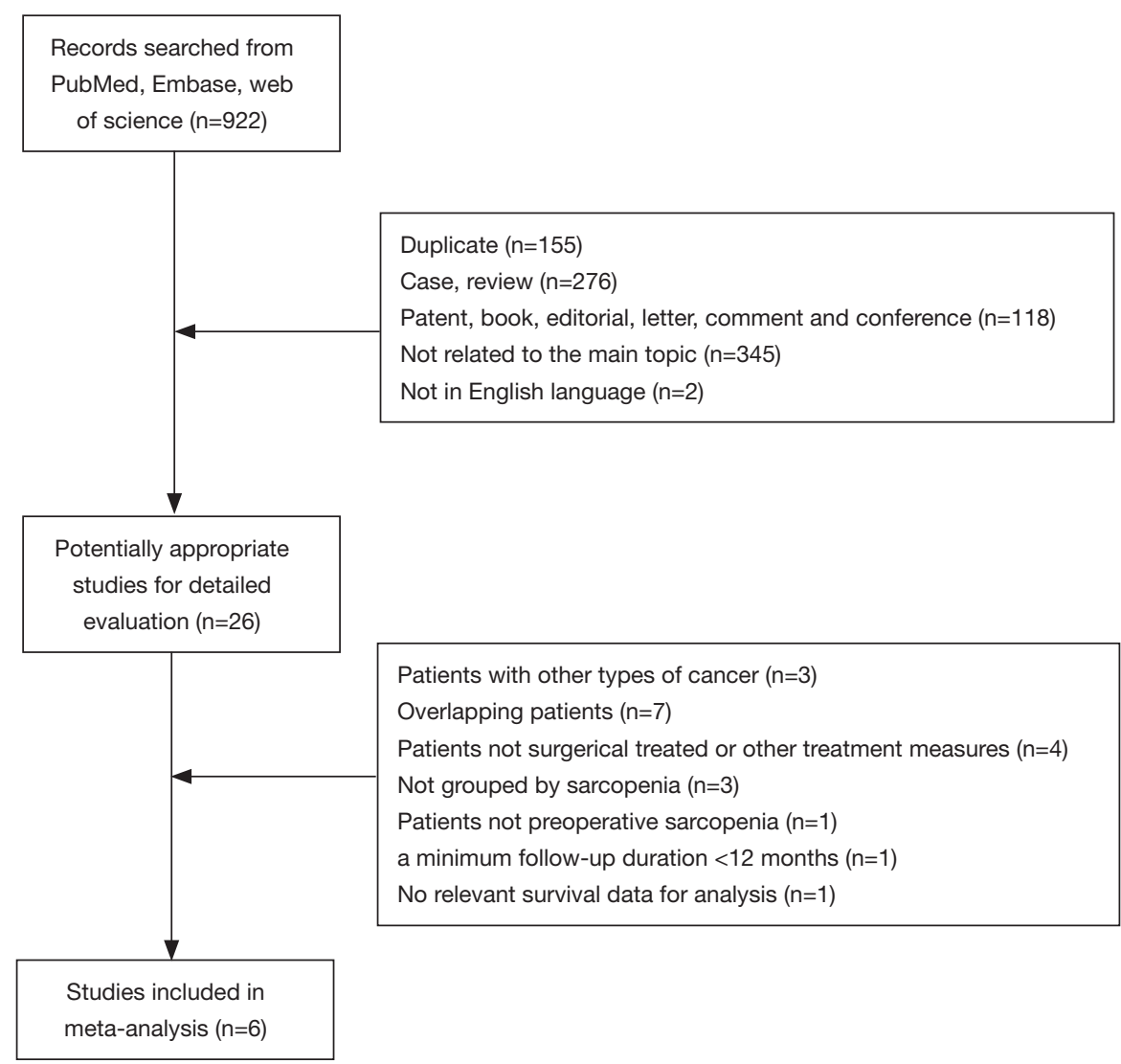

Figure 1 Flow chart of study selection based on the inclusion and exclusion criteria.

to measure skeletal muscle preoperatively and the level of skeletal muscle was measured in L3 in all studies. Two types of CT-assessed sarcopenia indexes were reported, including the SMI and IMAC. SMI was used to assess skeletal muscle quantity and was calculated by dividing the cross-sectional area of skeletal muscle in L3 by the height of the patient (in meters squared). IMAC was used to evaluate skeletal muscle quality and was calculated by dividing the CT value of the multifidus muscles [Hounsfield units (HU)] by the CT value of subcutaneous fat (HU). SMI was the most commonly used index and was utilized in all six studies $(17,18,21-23,26)$, with one study (26) employing both SMI and IMAC. The skeletal muscles used to calculate sarcopenia at the L3 level included psoas, paraspinal (erector spinae, multifidus, and quadratus lumborum), and abdominal wall muscles (transversus abdominus, external and internal obliques, and rectus abdominus). In various studies, different $\mathrm{HU}$ thresholds were used to identify and quantify skeletal muscle [-29 to $150(21-23,26),-29$ to 110 (18), and -30 to 110 (17)]. According to the calculation formula, a low SMI denoted low muscle mass, while a high IMAC signified low muscle quality. Internationally, accepted cut-off values or study-specific cut-offs were applied to define sarcopenia. International consensus cut-offs were adopted in four studies $(17,18,21,22)$. Data from 657 healthy cases of living donor liver transplantation (LDLT) were used in one study (26). Receiver operator characteristic (ROC) curves (20) and optimum stratification (23) were used in the other studies. Almost all of the included studies used sex-specific cut-offs and females had lower muscle mass and area compared to males. The NOS quality score ranged from 8 to 9 , as shown in Table 3.

\section{Sarcopenia and short-term prognosis}

Table 4 shows the relationship between sarcopenia and early postoperative prognosis. There were no significant differences in postoperative short-term outcomes between the groups with and without preoperative sarcopenia. The details were as follows: overall complications (OR 

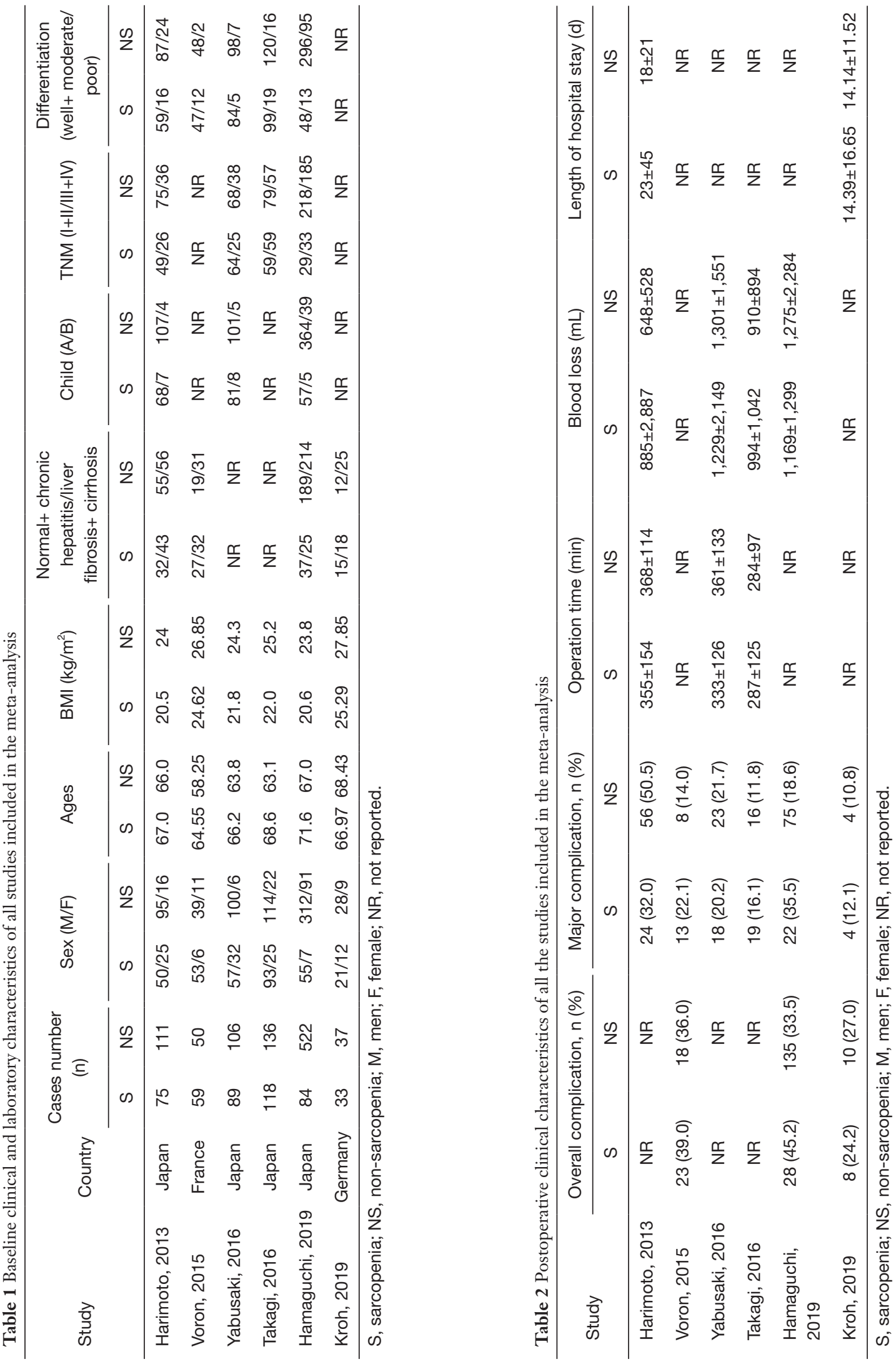
Table 3 Main characteristics of the included studies and results of the NOS quality assessment

\begin{tabular}{|c|c|c|c|c|c|c|c|}
\hline Study, year & Study design & Study period & NOS & $\begin{array}{l}\text { Modality } \\
\text { and level }\end{array}$ & Indexes & $\begin{array}{l}\text { The involved muscles and } \\
\text { HU chosen by CT scan }\end{array}$ & $\begin{array}{l}\text { Cut-off value for } \\
\text { sarcopenia }\end{array}$ \\
\hline Harimoto, 2013 & Retrospective & $\begin{array}{l}1 / 2004- \\
12 / 2009\end{array}$ & 8 & L3 & SMI & $\begin{array}{l}\text { All the skeletal muscle; }-29 \\
\text { and } 150 \mathrm{HU}\end{array}$ & $\begin{array}{l}\text { Males: } 43.75 \mathrm{~cm}^{2} / \mathrm{m}^{2} ; \\
\text { females: } 41.10 \mathrm{~cm}^{2} / \mathrm{m}^{2}\end{array}$ \\
\hline Voron, 2015 & Retrospective & 2/2006-9/2012 & 9 & L3 & SMI & $\begin{array}{l}\text { All the skeletal muscle; }-29 \\
\text { and } 150 \mathrm{HU}\end{array}$ & $\begin{array}{l}\text { Males: } 52.4 \mathrm{~cm}^{2} / \mathrm{m}^{2} \\
\text { females: } 38.9 \mathrm{~cm}^{2} / \mathrm{m}^{2}\end{array}$ \\
\hline Takagi, 2016 & Retrospective & $\begin{array}{l}1 / 2007- \\
12 / 2013\end{array}$ & 9 & L3 & SMI & $\begin{array}{l}\text { All the skeletal muscle; }-29 \\
\text { and } 150 \mathrm{HU}\end{array}$ & $\begin{array}{l}\text { Males: } 46.4 \mathrm{~cm}^{2} / \mathrm{m}^{2} ; \\
\text { females: } 37.6 \mathrm{~cm}^{2} / \mathrm{m}^{2}\end{array}$ \\
\hline Kroh, 2019 & Retrospective & $\begin{array}{l}1 / 2010- \\
12 / 2014\end{array}$ & 8 & L3 & SMI & $\begin{array}{l}\text { All the skeletal muscle; }-30 \\
\text { and } 110 \mathrm{HU}\end{array}$ & $\begin{array}{l}\text { Males: } 43 \mathrm{~cm}^{2} / \mathrm{m}^{2} \text { for BMI } \\
<25 \mathrm{~kg} / \mathrm{m}^{2}, 53 \mathrm{~cm}^{2} / \mathrm{m}^{2} \text { for } \\
\text { BMI > } 25 \mathrm{~kg} / \mathrm{m}^{2} ; \\
\text { females: } 41 \mathrm{~cm}^{2} / \mathrm{m}^{2}\end{array}$ \\
\hline $\begin{array}{l}\text { Hamaguchi, } \\
2019\end{array}$ & & & & & IMAC & The multifidus muscle; NR & $\begin{array}{l}\text { Males: }-0.358 \\
\text { females: }-0.229\end{array}$ \\
\hline
\end{tabular}

NOS, Newcastle-Ottawa Scale; SMI, skeletal muscle mass index; IMAC, intramuscular adipose tissue content.

Table 4 A meta-analysis of the association between sarcopenia and early postoperative prognosis

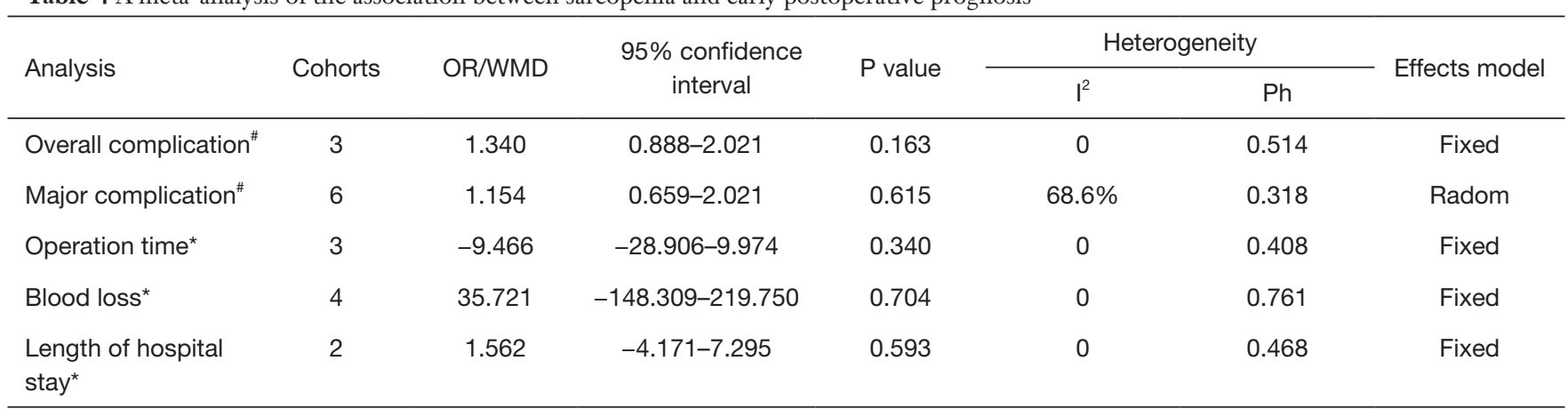

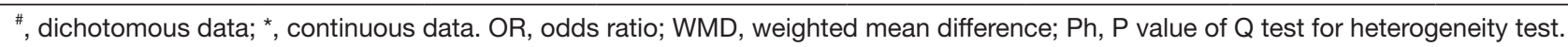

$=1.340,95 \%$ CI: $0.888-2.021, \mathrm{P}=0.163, \mathrm{I}^{2}=0, \mathrm{Ph}=0.514$, Table 4, Figure 2A), major complications $(\mathrm{OR}=1.154$, 95\% CI: 0.659-2.021, $\mathrm{P}=0.615, \mathrm{I}^{2}=68.6 \%, \mathrm{Ph}=0.318$, Table 4, Figure $2 \mathrm{~B})$, operation time $(\mathrm{WMD}=-9.466$, 95\% CI: $-28.906-9.974, \mathrm{P}=0.340, \mathrm{I}^{2}=0, \mathrm{Ph}=0.408$, Table 4, Figure 2C), estimated blood loss (WMD $=35.721$, 95\% CI: $-148.309-219.750, \mathrm{P}=0.704, \mathrm{I}^{2}=0, \mathrm{Ph}=0.761$, Table 4 , Figure 2D), and length of hospital stay $(\mathrm{WMD}=1.562$, 95\% CI: -4.171-7.295, $\mathrm{P}=0.593, \mathrm{I}^{2}=0, \mathrm{Ph}=0.468$, Table 4, Figure 2E). A random effects model was only employed for major complications, and a fixed effects model was employed for the remaining variables.

\section{Sarcopenia and long-term prognosis}

\section{Assessment of overall survival}

Seven cohorts in six studies reported the effect of preoperative muscle reduction on the overall survival rate of HCC patients treated with surgery, with 1,420 patients in total (458 sarcopenic and 962 non-sarcopenic patients). The meta-analysis showed that patients with sarcopenia had a poor OS, with a pooled HR of 1.572 (95\% CI: 1.342-1.840, $\mathrm{P}=0.000$, Table 5, Figure $3 A$ ), and a fixed-effects model was employed in these studies due to a lack of heterogeneity $\left(\mathrm{I}^{2}=31.7 \%, \mathrm{Ph}=0.186\right)$. In the stratified analyses by SMI, 


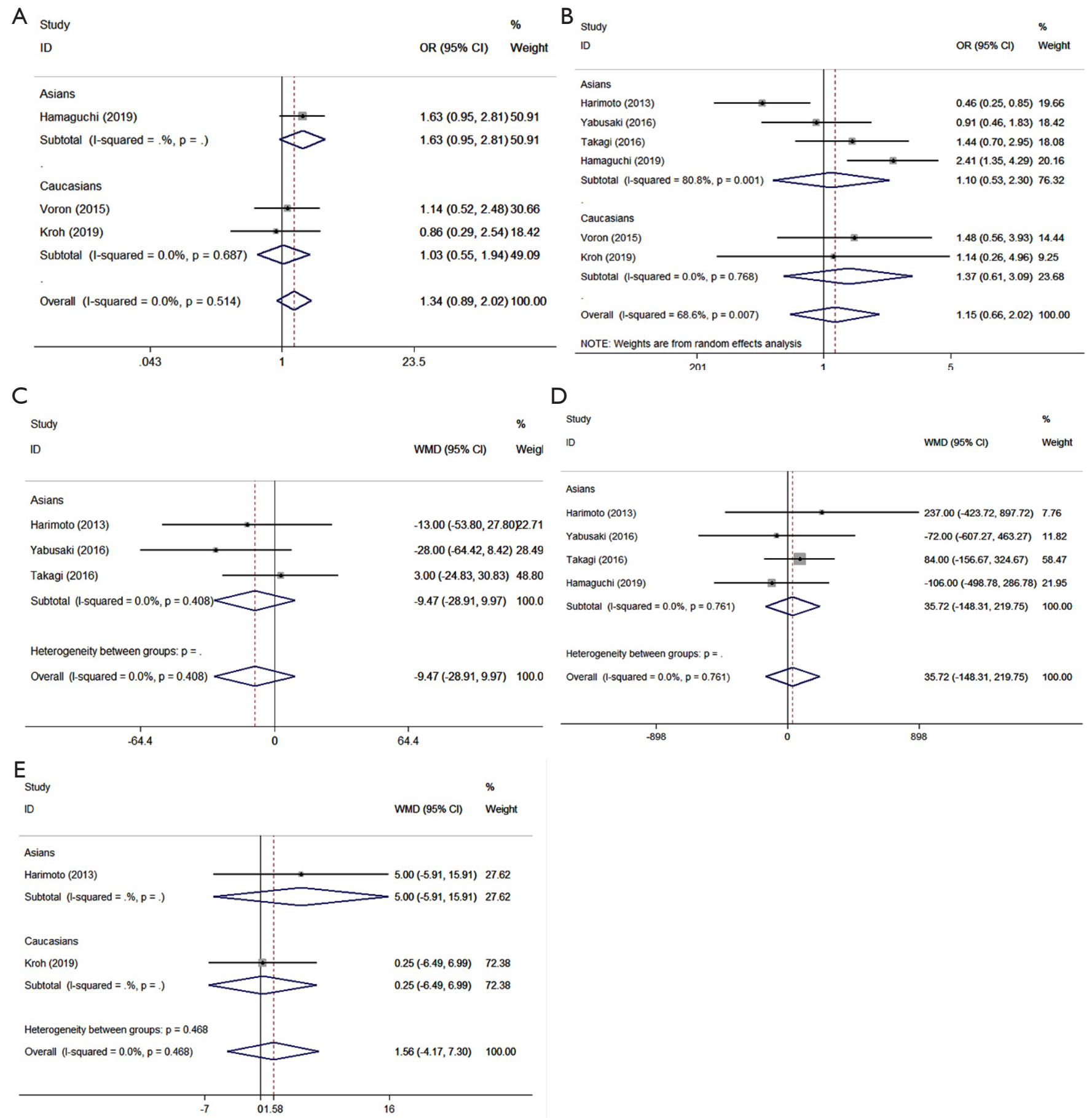

Figure 2 Forest plot of OR/WMD for the association between sarcopenia and early postoperative prognosis in patients with surgically treated HCC. (A) Overall complications; (B) major complications; (C) operation time; (D) estimated blood loss; (E) length of hospital stay. OR, odds ratio; WMD, weighted mean difference; HCC, hepatocellular carcinoma. 
Table 5 A meta-analysis of the association between sarcopenia and long-term prognosis outcomes

\begin{tabular}{|c|c|c|c|c|c|c|c|c|c|}
\hline Analysis & Cohorts & HR & $\begin{array}{l}95 \% \text { confidence } \\
\text { interval }\end{array}$ & $P$ value & \multicolumn{2}{|c|}{ Heterogeneity } & $\begin{array}{l}\text { Effects } \\
\text { model }\end{array}$ & $\begin{array}{l}\text { Egger's test } \\
\mathrm{P} \text { value }\end{array}$ & $\begin{array}{l}\text { Begg's test } \\
\mathrm{P} \text { value }\end{array}$ \\
\hline OS & 7 & 1.572 & $1.342-1.840$ & $0^{\wedge}$ & $31.7 \%$ & 0.186 & Fixed & 0.548 & 0.523 \\
\hline SMI & 6 & 1.462 & $1.198-1.784$ & $0^{\wedge}$ & $32.7 \%$ & 0.191 & & & \\
\hline Asians & 4 & 1.410 & $1.136-1.751$ & $0.002^{\wedge}$ & $33.0 \%$ & 0.214 & & & \\
\hline IMAC & 1 & 1.774 & $1.371-2.295$ & $0^{\wedge}$ & - & - & & & \\
\hline DFS & 4 & 1.544 & $1.178-2.024$ & $0.002^{\wedge}$ & $64.1 \%$ & 0.039 & Random & 0.089 & $0.018^{\wedge}$ \\
\hline SMI & 3 & 1.744 & $1.225-2.484$ & $0.002^{\wedge}$ & $59.2 \%$ & 0.086 & & & \\
\hline Asians & 2 & 1.487 & $1.189-1.860$ & $0.001^{\wedge}$ & 0 & 0.768 & & & \\
\hline
\end{tabular}

OS, overall survival; SMI, skeletal muscle mass index; IMAC, intramuscular adipose tissue content; DFS, disease-free survival; HR, hazard ratio; $\mathrm{Ph}, \mathrm{P}$ value of $\mathrm{Q}$ test for heterogeneity test. $\wedge \mathrm{P}<0.05$.

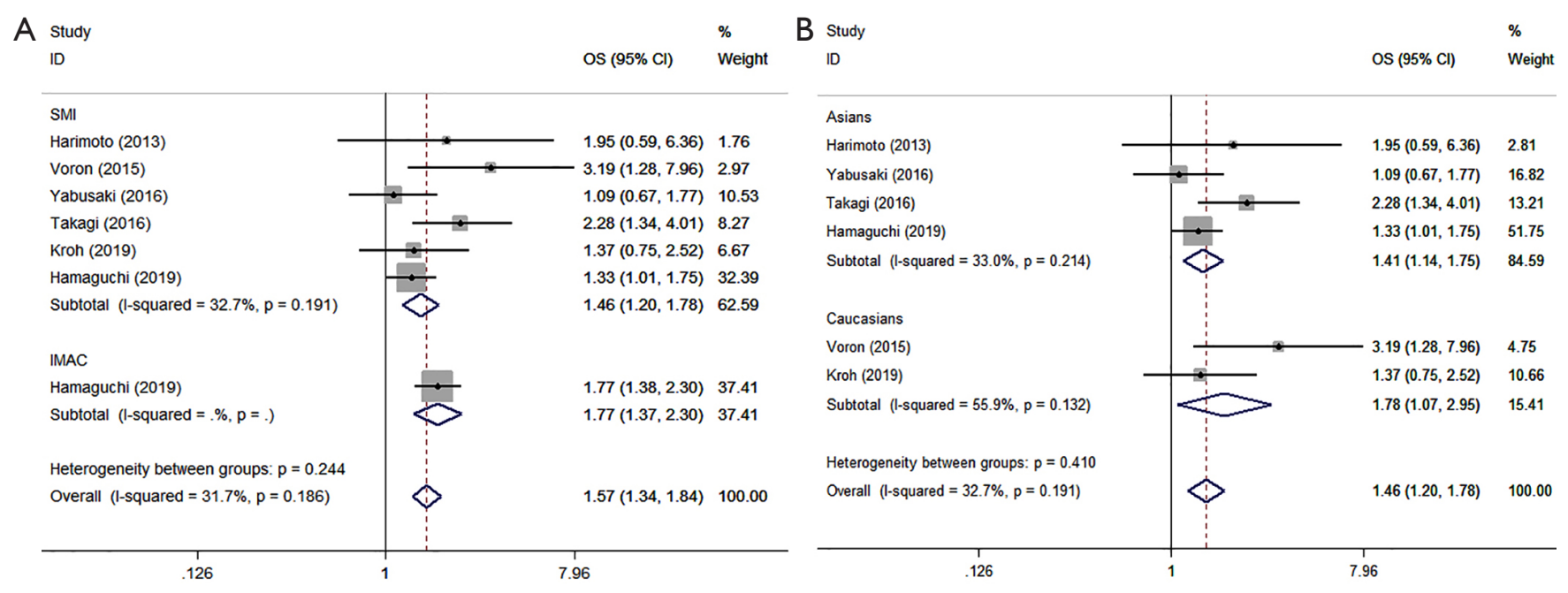

Figure 3 Forest plot of HR for the association between preoperative sarcopenia and OS in patients with surgically treated HCC. (A) Subgroup analyses stratified by types of CT-assessed sarcopenia indexes (SMI/IMAC); (B) the stratified analyses by ethnicity. HR, hazard ratio; OS, overall survival; HCC, hepatocellular carcinoma.

the same conclusion was reached $(\mathrm{HR}=1.462,95 \% \mathrm{CI}$ : $1.198-1.784, \mathrm{P}=0.000)$, with $\mathrm{I}^{2}=32.7 \%$ and $\mathrm{Ph}=0.191$ (Table 5, Figure $3 A$ ). It is worth noting that the same conclusions were made when conducting the subgroup analyses stratified by ethnicity (Asians: $\mathrm{HR}=1.410,95 \% \mathrm{CI}$ : 1.136-1.751, $\mathrm{P}=0.002$, with $\mathrm{I}^{2}=33.0 \% \mathrm{Ph}=0.214$; Caucasians: HR $=1.779,95 \%$ CI: $1.071-2.954, \mathrm{P}=0.026$, with $\mathrm{I}^{2}=55.9 \%$, $\mathrm{Ph}=0.132$, Table 5, Figure 3B).

\section{Assessment of disease-free survival}

Four cohorts in six studies reported data regarding the association between preoperative sarcopenia and DFS in 901 patients (218 sarcopenic patients and 683 nonsarcopenic patients). Using a random effects model, the meta-analysis revealed that preoperative sarcopenia was significantly associated with shorter DFS (HR $=1.544,95 \%$ CI: 1.178-2.024, $\mathrm{P}=0.002$, Table 5, Figure $4 A$ ), with observed 


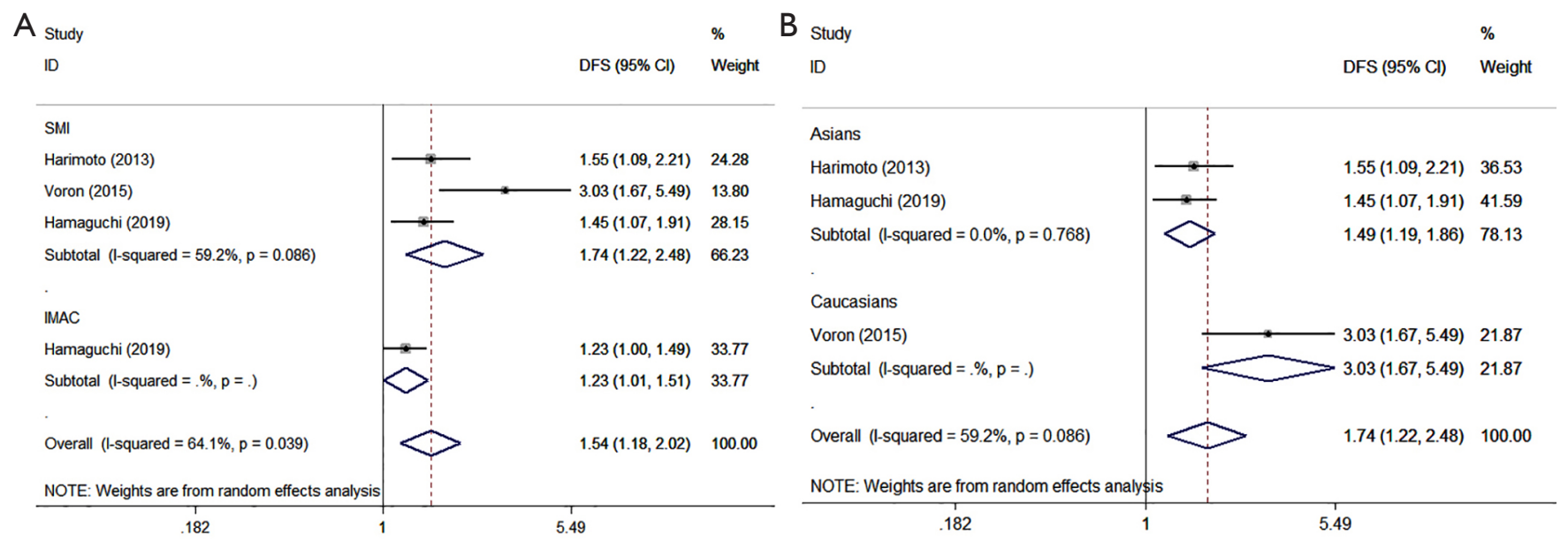

Figure 4 Forest plot of HR for the association between preoperative sarcopenia and DFS in patients with surgically treated HCC. (A) Subgroup analyses stratified by types of CT-assessed sarcopenia indexes (SMI/IMAC); (B) the stratified analyses by ethnicity. HR, hazard ratio; DFS, disease-free survival; HCC, hepatocellular carcinoma; SMI, skeletal muscle mass index; IMAC, intramuscular adipose tissue content.

Table 6 A meta-analysis of the association between sarcopenia and clinical pathological factors

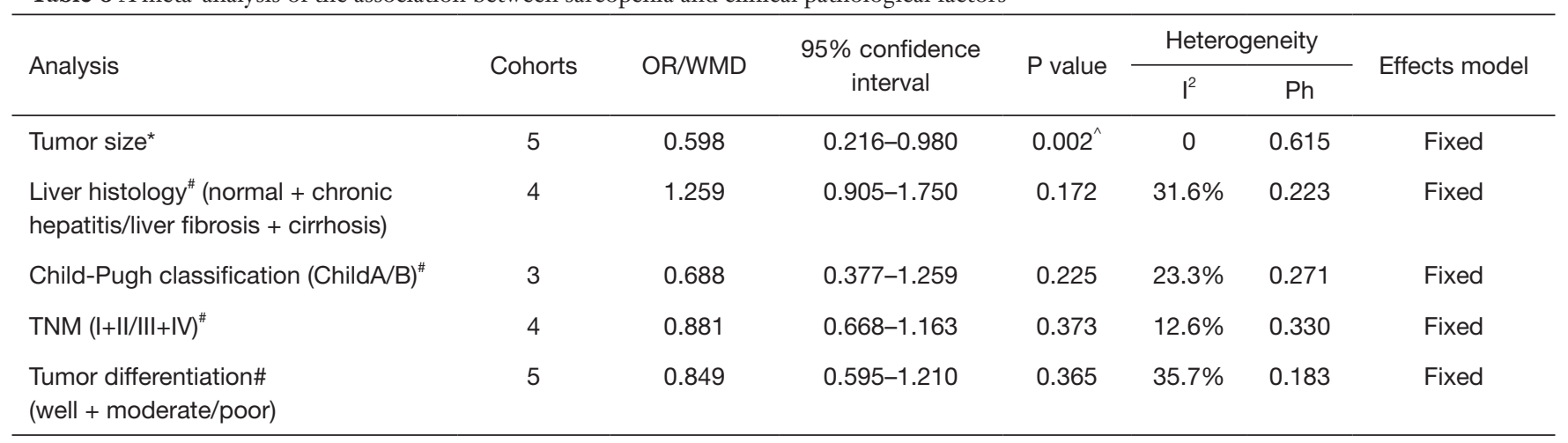

\#, dichotomous data; ${ }^{*}$, continuous data. ${ }^{\wedge} \mathrm{P}<0.05$. OR, odds ratio; WMD, weighted mean difference; Ph, $\mathrm{P}$ value of $\mathrm{Q}$ test for heterogeneity test.

heterogeneity $\left(\mathrm{I}^{2}=64.1 \%, \mathrm{Ph}=0.039\right)$. The same conclusion was made in the stratified analyses by SMI representing muscle quantity (HR $=1.744,95 \%$ CI: 1.225-2.484, $\mathrm{P}=0.002$, Table 5, Figure $4 A$ ), with $\mathrm{I}^{2}=59.2 \%$ and $\mathrm{Ph}=0.086$. It is noteworthy that the same conclusions were made when performing the subgroup analyses stratified by ethnicity (Asian: HR $=1.487,95 \%$ CI: $1.189-1.860, \mathrm{P}=0.001$, with $\mathrm{I}^{2}=0 \%, \mathrm{Ph}=0.768$; Caucasians: $\mathrm{HR}=3.030,95 \% \mathrm{CI}: 1.671-$ 5.494, $\mathrm{P}=0$, Table 5, Figure 4B).

\section{Sarcopenia and clinicopatbological factors}

The auxiliary result of this meta-analysis was the relationship between preoperative sarcopenia and clinicopathological factors. Patients with preoperative sarcopenia (Asian subjects in particular) tended to have larger diameter tumors (WMD $=0.598,95 \% \mathrm{CI}$ : $0.216-0.980, \mathrm{P}=0.002, \mathrm{I}^{2}=0, \mathrm{Ph}=0.615$, Table 6, Figure $5 A$ ). In the meta-analysis assessing the association between preoperative sarcopenia and liver histology $(\mathrm{OR}=1.259$, 95\% CI: $0.905-1.750, \mathrm{P}=0.172, \mathrm{I}^{2}=31.6 \%, \mathrm{Ph}=0.223$, Table 6, Figure 5B), Child-Pugh classification $(\mathrm{OR}=0.688$, 95\% CI: $0.337-1.259, \mathrm{P}=0.225, \mathrm{I}^{2}=23.3 \%, \mathrm{Ph}=0.271$, Table 6, Figure 5C), and TNM stage (OR $=0.881,95 \%$ CI: $0.668-1.163, \mathrm{P}=0.373, \mathrm{I}^{2}=12.6 \%, \mathrm{Ph}=0.330$, Table 6, Figure $5 D$ ), the results failed to show statistical significance. 
A

Study ID

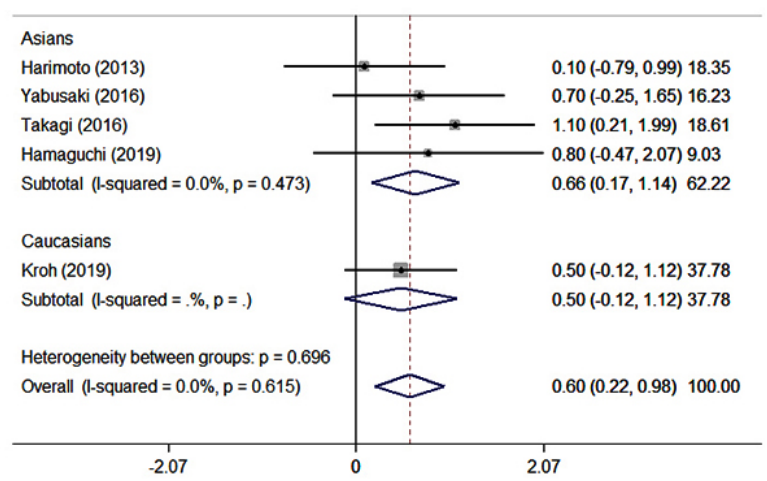

C

Study

ID

$\%$ WMD $(95 \%$ Cl) Weight

\section{$0.10(-0.79,0.99) 18.35$} $(-0.25,1.65) 16.23$ $0.80(-0.47,2.07) 9.03$

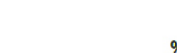

OR $(95 \% \mathrm{Cl}) \quad$ Weight

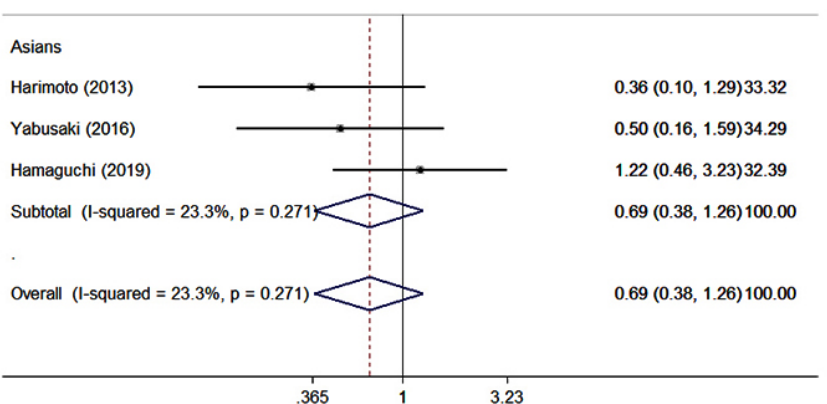

B Study $\%$ ID $\quad$ OR $(95 \% \mathrm{Cl}) \quad$ Weight

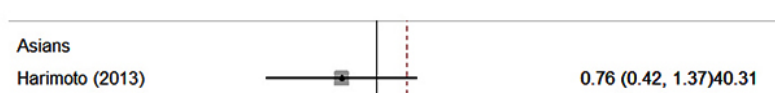
$\begin{array}{llll}\text { Hamaguchi (2019) } & & 1.68(0.97,2.89) 32.22\end{array}$ Subtotal (l-squared $=73.4 \%, \mathrm{p}=0.052) \longrightarrow 1.17(0.79,1.73) 72.53$

Caucasians

Voron (2015) Kroh (2019) Subtotal (l-squared $=0.0 \%, p=0.713$ ) Overall (I-squared $=31.5 \%, p=0.223$ ) 218

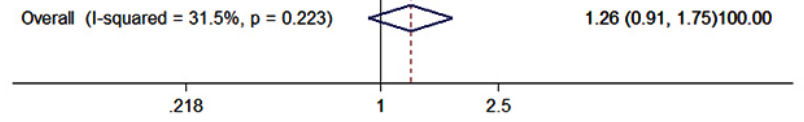

D Study ID

$\mathrm{OR}(95 \% \mathrm{Cl})$ $\%$

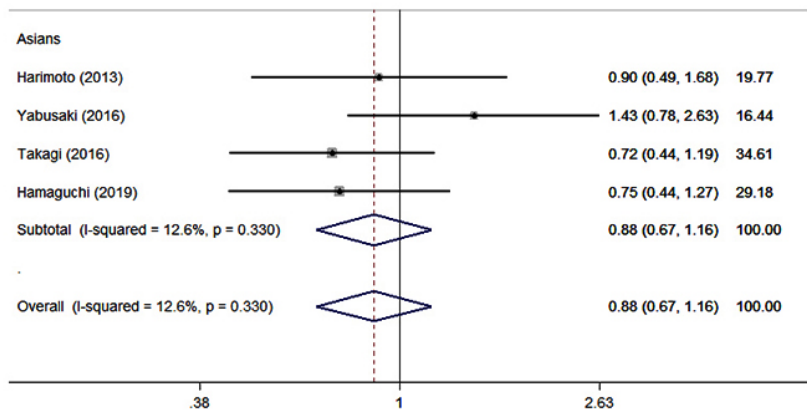

$E_{\text {sudy }}$

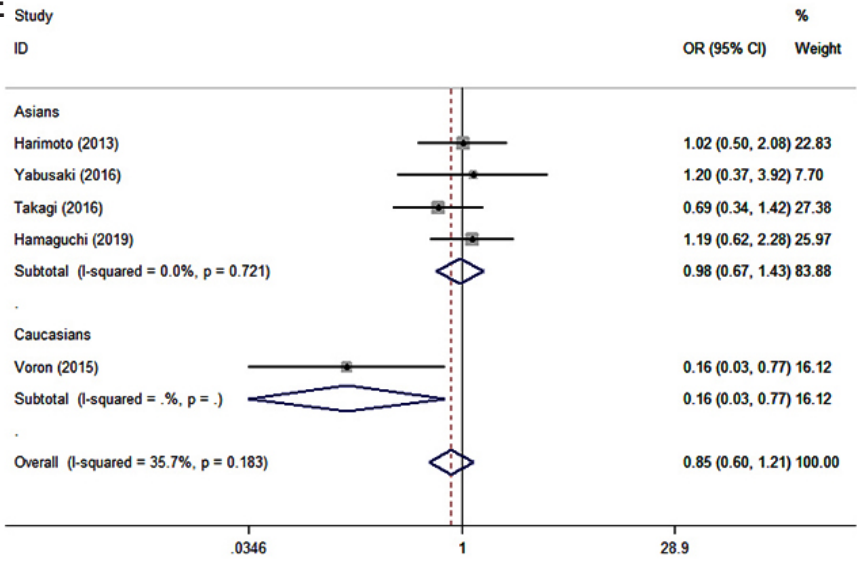

Figure 5 Forest plot of OR/WMD for the association between preoperative sarcopenia and the clinicopathological factors in patients with surgically treated HCC. (A) Tumor sizes; (B) liver histology; (C) Child-Pugh classification; (D) TNM; (E) tumor differentiation. OR, odds ratio; WMD, weighted mean difference; HCC, hepatocellular carcinoma. 

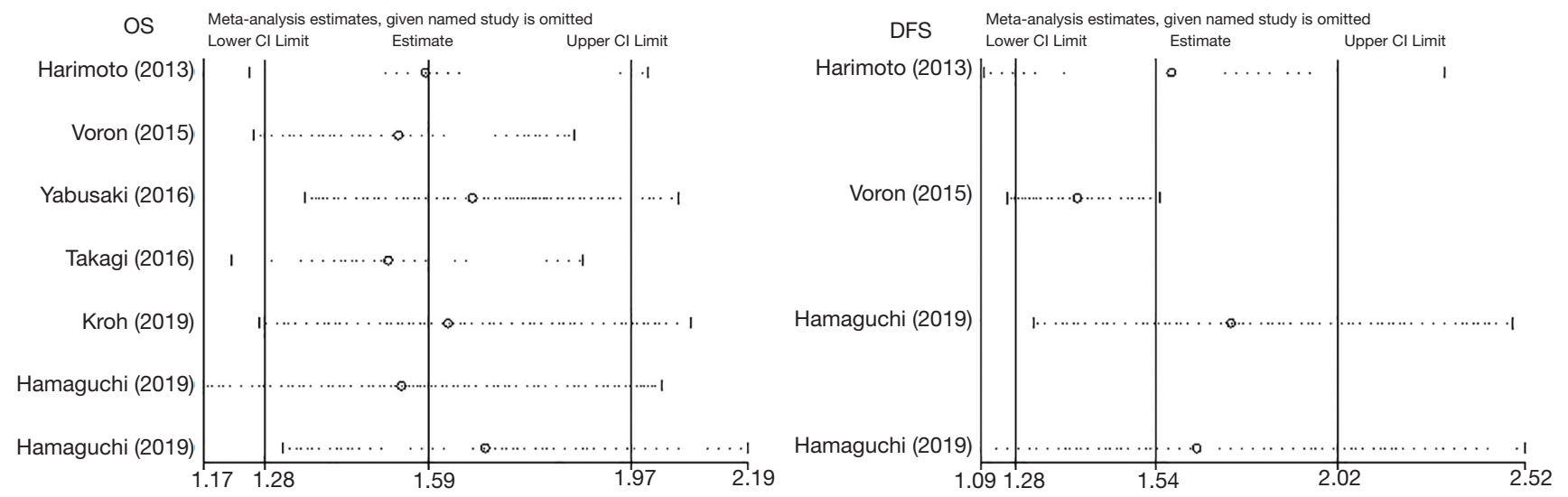

Figure 6 Sensitivity analysis for overall survival (OS) and disease-free survival (DFS).

The association between preoperative sarcopenia and tumor differentiation was not observed $(\mathrm{OR}=0.849,95 \%$ CI: $0.595-1.210, \mathrm{P}=0.365, \mathrm{I}^{2}=35.7 \%, \mathrm{Ph}=0.183$, Table 6, Figure 5E). Though, it is worth noting that Caucasian patients with preoperative sarcopenia had a greater likelihood of malignancy ( $\mathrm{OR}=0.163,95 \%$ CI: $0.035-0.769$, $\mathrm{P}=0.022$, Figure $5 E$ ). No heterogeneity was observed, and a fixed-effects model was employed for all comparisons.

\section{Sensitivity analysis and publication bias}

A sensitivity analysis was used to evaluate the stability of our results based on OS and DFS by successively removing each study. The results demonstrated that no individual study qualitatively changed the pooled HRs, indicating that our results were robust (Figure 6). Egger's test and Begg's test were utilized to assess publication bias. The Egger's test did not indicate any evidence of publication bias between preoperative sarcopenia and long-term prognosis (OS: $\mathrm{P}=0.548$; DFS: $\mathrm{P}=0.089$ ). This result was confirmed by the Begg's test for OS $(\mathrm{P}=0.523)$, though, not for DFS $(\mathrm{P}=0.018)$ (Table 5).

\section{Discussion}

Based on its etiology, sarcopenia can be divided into primary and secondary sarcopenia (27). Primary sarcopenia, also known as age-related sarcopenia, is fundamentally caused by aging. In contrast, secondary sarcopenia is accompanied by a variety of diseases, such as inflammatory disease, malignancy, endocrine disease, malnutrition, or advanced organ function failures (e.g., heart, lung, kidney, liver, and brain). So far, a variety of imaging methods, including dual-energy X-ray absorptiometry (DXA), bioelectrical impedance analysis (BIA), computed tomography (CT), magnetic resonance (MR) and ultrasound (US), have been used to evaluate muscle quality and quantity and to diagnose sarcopenia $(28,29)$. With the conventional advantage of staging and follow-up of cancer, CT offers the best opportunity to evaluate sarcopenia without the need for additional testing. In contrast to DXA, which provides a whole-body estimate of muscle mass, CT measures muscle size and decay in specific areas (30). Thus, CT has been used to assess the composition of the body in some retrospective and prospective analyses. Even with CT alone, various indicators have been used in different studies to define sarcopenia (31). According to the latest research, CT-defined sarcopenia can be divided into two categories based on the quantity and the quality of the muscles. In our study, SMI represented muscle quantity, while IMAC was related to muscle quality. Among the muscle quantity indexes, SMI $\left(\mathrm{cm}^{2} / \mathrm{m}^{2}\right)$ was equal to the cross-sectional area $\left(\mathrm{cm}^{2}\right)$ of all skeletal muscle in the $\mathrm{L} 3$ region on CT images divided by the square of body height $\left(\mathrm{m}^{2}\right)$ (32). SMI shows excellent consistency with DXA scanning on muscle mass (33) and is presently a widely-used skeletal muscle quantity assessment method. Among the muscle quality indexes, IMAC was equal to the CT value (HU) of the multifidus muscles divided by the CT value (HU) of the subcutaneous fat $(19,20)$.

CT-defined sarcopenia indexes have been reported in several studies as strong and independent prognostic factors for poor postoperative surgical and oncology treatment outcomes in patients with some diseases and types of cancer, particularly in digestive tract cancers (11-14,34-37). However, to date, the conclusions drawn from different 
indicators and studies remain controversial $(17,38)$. For instance, Chang et al. (37) conducted a meta-analysis of the relationship between the loss of skeletal muscle mass and survival in patients with HCC in March 2018. However, the included patients in that study had used treatments other than (or in addition to) surgery, including sorafenib, ablation therapy, transcatheter arterial chemoembolization (TACE), and even liver transplantation. As far as we know, the treatments above can affect the outcome of HCC patients. Thus, in order to obtain a more accurate estimation of the relationship between preoperative sarcopenia and the prognosis of patients with surgically treated HCC, we performed this meta-analysis.

In this paper, a meta-analysis of six studies, including 1,420 patients, was conducted to determine the predictive value of CT-determined sarcopenia for the prognosis of HCC patients after hepatectomy. The results showed that preoperative skeletal muscle loss was a powerful predictor of OS and DFS in HCC patients, especially in patients with sarcopenia measured by L3-SMI. Additionally, preoperative sarcopenia was also significantly associated with tumor size, and patients (especially Asians) with sarcopenia tended to have larger diameter tumors than those without sarcopenia, though Caucasian patients with sarcopenia did not exhibit this trend. We hypothesized that the reasons for this disparity were the differences in the cut-off values for sarcopenia and fewer enrolled patients.

It is worth noting that most of the $\mathrm{I}^{2}$ values in this study were quite low, indicating that the heterogeneity can be ignored, further improving the reliability of the results. In fact, due to the nature of the prognosis analysis of sarcopenia, most of the included study designs were similar, especially in terms of participant enrolment and CT image analysis methods in the original literature. This consistency in research designs may account for the lack of heterogeneity. However, there was considerable heterogeneity in the study conducted by Voron et al. (21) and Kroh et al. (17) in Caucasian subjects (Table 5).

In conclusion, our meta-analysis provided powerful evidence to evaluate the effect of preoperative sarcopenia on the prognosis of patients with HCC after surgery. However, our study has some limitations that should be noted. Firstly, a consensus on the cut-offs for CT-assessed sarcopenia indexes and $\mathrm{HU}$ values of involved muscles chosen by CT scan were still lacking in the included studies; though, we performed subgroup analyses based on different sarcopenia indexes and ethnicity. Secondly, retrospective studies did not consider muscle strength and function. Thirdly, some adjuvant therapies, such as ablation therapy, TACE, and lipiodolization, are essential factors affecting the prognosis of HCC patients. However, the studies we included were not well documented. Fourthly, we were unable to perform subgroup analysis based on the primary location of the tumor (such as the right or left liver) and the presence of a cancer embolus in the portal vein. Lastly, the HRs were not described directly or calculated according to survival data or survival curves in the original articles. We attempted to e-mail the corresponding authors. However, we were still unable to obtain survival data. These limitations affect our ability to understand the relationship between preoperative sarcopenia and postoperative prognosis, and especially long-term prognosis, in patients with HCC after surgery. Even if we get detailed data, such as postoperative adjuvant therapy, primary location of the tumor and cancer embolus in the portal vein, the interpretation and the comparison of survival data from different published studies should be taken with caution. Thus, further large-scale prospective studies are needed to confirm our findings. And an individual patient data approach which combine all data from the individual papers in one dataset is also in our consideration.

\section{Acknowledgments}

Funding: Supported by National Key R\&D Program of China (2018YFC2000301) and National Key R\&D Program of China (2018YFC2001904).

\section{Footnote}

Reporting Checklist: The authors present the study in accordance with the PRISMA reporting checklist. Available at: http://dx.doi.org/10.21037/jgo-20-122

Data Sharing Statement: Available at http://dx.doi. org/10.21037/jgo-20-122

Conflicts of Interest: All authors have completed the ICMJE uniform disclosure form (available at: http://dx.doi. org/10.21037/jgo-20-122). The authors have no conflicts of interest to declare.

Ethical Statement: The authors are accountable for all aspects of the work in ensuring that questions related to the accuracy or integrity of any part of the work are appropriately investigated and resolved. This study was 
a systematic review and meta-analysis of all published literature and institutional review deemed this study exempt from ethical approval.

Open Access Statement: This is an Open Access article distributed in accordance with the Creative Commons Attribution-NonCommercial-NoDerivs 4.0 International License (CC BY-NC-ND 4.0), which permits the noncommercial replication and distribution of the article with the strict proviso that no changes or edits are made and the original work is properly cited (including links to both the formal publication through the relevant DOI and the license). See: https://creativecommons.org/licenses/by-nc-nd/4.0/.

\section{References}

1. Torre LA, Bray F, Siegel RL, et al. Global cancer statistics, 2012. CA Cancer J Clin 2015;65:87-108.

2. Hammad A, Kaido T, Ogawa K, et al. Liver transplantation for advanced hepatocellular carcinoma in patients with Child-Pugh A and B. Surg Today 2016;46:248-54.

3. Fang Q, Xie QS, Chen JM, et al. Long-term outcomes after hepatectomy of huge hepatocellular carcinoma: A single-center experience in China. Hepatobiliary Pancreat Dis Int 2019;18:532-7.

4. Colecchia A, Schiumerini R, Cucchetti A, et al. Prognostic factors for hepatocellular carcinoma recurrence. World J Gastroenterol 2014;20:5935-50.

5. Lurje I, Czigany Z, Bednarsch J, et al. Treatment Strategies for Hepatocellular Carcinoma ${ }^{-}$a Multidisciplinary Approach. Int J Mol Sci 2019;20:1465.

6. Kim G, Kang SH, Kim MY, et al. Prognostic value of sarcopenia in patients with liver cirrhosis: A systematic review and meta-analysis. PLoS One 2017;12:e0186990.

7. Bekfani T, Pellicori P, Morris DA, et al. Sarcopenia in patients with heart failure with preserved ejection fraction: Impact on muscle strength, exercise capacity and quality of life. Int J Cardiol 2016;222:41-6.

8. Marty E, Liu Y, Samuel A, et al. A review of sarcopenia: Enhancing awareness of an increasingly prevalent disease. Bone 2017;105:276-86.

9. Cruz-Jentoft AJ, Baeyens JP, Bauer JM, et al. Sarcopenia: European consensus on definition and diagnosis: Report of the European Working Group on Sarcopenia in Older People. Age Ageing 2010;39:412-23.

10. Ha Y, Kim D, Han S, et al. Sarcopenia Predicts Prognosis in Patients with Newly Diagnosed Hepatocellular Carcinoma, Independent of Tumor Stage and Liver
Function. Cancer Res Treat 2018;50:843-51.

11. Deng HY, Zha P, Peng L, et al. Preoperative sarcopenia is a predictor of poor prognosis of esophageal cancer after esophagectomy: a comprehensive systematic review and meta-analysis. Dis Esophagus 2019;32:doy115.

12. Nishimura JM, Ansari AZ, D'Souza DM, et al. Computed Tomography-Assessed Skeletal Muscle Mass as a Predictor of Outcomes in Lung Cancer Surgery. Ann Thorac Surg 2019;108:1555-64.

13. Kamarajah SK, Bundred J, Tan BHL. Body composition assessment and sarcopenia in patients with gastric cancer: a systematic review and meta-analysis. Gastric Cancer 2019;22:10-22.

14. Hahn F, Muller L, Stohr F, et al. The role of sarcopenia in patients with intrahepatic cholangiocarcinoma: Prognostic marker or hyped parameter? Liver Int 2019;39:1307-14.

15. Fujiwara N, Nakagawa H, Kudo Y, et al. Sarcopenia, intramuscular fat deposition, and visceral adiposity independently predict the outcomes of hepatocellular carcinoma. J Hepatol 2015;63:131-40.

16. Kobayashi A, Kaido T, Hamaguchi Y, et al. Impact of Sarcopenic Obesity on Outcomes in Patients Undergoing Hepatectomy for Hepatocellular Carcinoma. Ann Surg 2019;269:924-31.

17. Kroh A, Uschner D, Lodewick T, et al. Impact of body composition on survival and morbidity after liver resection in hepatocellular carcinoma patients. Hepatobiliary Pancreat Dis Int 2019;18:28-37.

18. Yabusaki N, Fujii T, Yamada S, et al. Adverse impact of low skeletal muscle index on the prognosis of hepatocellular carcinoma after hepatic resection. Int J Surg 2016;30:136-42.

19. Hamaguchi Y, Kaido T, Okumura S, et al. Muscle Steatosis is an Independent Predictor of Postoperative Complications in Patients with Hepatocellular Carcinoma. World J Surg 2016;40:1959-68.

20. Hamaguchi Y, Kaido T, Okumura S, et al. Preoperative intramuscular adipose tissue content is a novel prognostic predictor after hepatectomy for hepatocellular carcinoma. J Hepatobiliary Pancreat Sci 2015;22:475-85.

21. Voron T, Tselikas L, Pietrasz D, et al. Sarcopenia Impacts on Short- and Long-term Results of Hepatectomy for Hepatocellular Carcinoma. Ann Surg 2015;261:1173-83.

22. Harimoto N, Shirabe K, Yamashita YI, et al. Sarcopenia as a predictor of prognosis in patients following hepatectomy for hepatocellular carcinoma. Br J Surg 2013;100:1523-30.

23. Takagi K, Yagi T, Yoshida R, et al. Sarcopenia and American Society of Anesthesiologists Physical Status in 
the Assessment of Outcomes of Hepatocellular Carcinoma Patients Undergoing Hepatectomy. Acta Med Okayama 2016;70:363-70.

24. Parmar MK, Torri V, Stewart L. Extracting summary statistics to perform meta-analyses of the published literature for survival endpoints. Stat Med 1998;17:2815-34.

25. Tierney JF, Stewart LA, Ghersi D, et al. Practical methods for incorporating summary time-to-event data into metaanalysis. Trials 2007;8:16.

26. Hamaguchi Y, Kaido T, Okumura S, et al. Preoperative Visceral Adiposity and Muscularity Predict Poor Outcomes after Hepatectomy for Hepatocellular Carcinoma. Liver Cancer 2019;8:92-109.

27. Berger MJ, Doherty TJ. Sarcopenia: prevalence, mechanisms, and functional consequences. Interdiscip Top Gerontol 2010;37:94-114.

28. Albano D, Messina C, Vitale J, et al. Imaging of sarcopenia: old evidence and new insights. Eur Radiol 2020;30:2199-208.

29. Sergi G, Trevisan C, Veronese N, et al. Imaging of sarcopenia. Eur J Radiol 2016;85:1519-24.

30. Lenchik L, Boutin RD. Sarcopenia: Beyond Muscle Atrophy and into the New Frontiers of Opportunistic Imaging, Precision Medicine, and Machine Learning. Semin Musculoskelet Radiol 2018;22:307-22.

31. Cao Q, Xiong Y, Zhong Z, et al. Computed TomographyAssessed Sarcopenia Indexes Predict Major Complications following Surgery for Hepatopancreatobiliary Malignancy: A Meta-Analysis. Ann Nutr Metab 2019;74:24-34.

Cite this article as: $\mathrm{Xu} \mathrm{L}$, Jing $\mathrm{Y}$, Zhao C, Zhang Q, Zhao X, Yang J, Wu L, Yang Y. Preoperative computed tomographyassessed skeletal muscle index is a novel prognostic factor in patients with hepatocellular carcinoma following hepatectomy: a meta-analysis. J Gastrointest Oncol 2020;11(5):1040-1053. doi: 10.21037/jgo-20-122
32. Kubo N, Kawanaka H, Hiroshige S, et al. Sarcopenia discriminates poor prognosis in elderly patients following emergency surgery for perforation panperitonitis. Ann Gastroenterol Surg 2019;3:630-7.

33. Holt DQ, Strauss BJ, Lau KK, et al. Body composition analysis using abdominal scans from routine clinical care in patients with Crohn's Disease. Scand J Gastroenterol 2016;51:842-7.

34. Jones K, Gordon-Weeks A, Coleman C, et al. Radiologically Determined Sarcopenia Predicts Morbidity and Mortality Following Abdominal Surgery: A Systematic Review and Meta-Analysis. World J Surg 2017;41:2266-79.

35. Cai C, Song X, Chen Y, et al. Relationship between relative skeletal muscle mass and nonalcoholic fatty liver disease: a systematic review and meta-analysis. Hepatol Int 2020;14:115-26.

36. Chang KV, Chen JD, Wu WT, et al. Association of loss of muscle mass with mortality in liver cirrhosis without or before liver transplantation: A systematic review and metaanalysis. Medicine (Baltimore) 2019;98:e14373.

37. Chang KV, Chen JD, Wu WT, et al. Association between Loss of Skeletal Muscle Mass and Mortality and Tumor Recurrence in Hepatocellular Carcinoma: A Systematic Review and Meta-Analysis. Liver Cancer 2018;7:90-103.

38. Nakayama N, Nakayama K, Nakamura K, et al. Sarcopenic Factors May Have No Impact on Outcomes in Ovarian Cancer Patients. Diagnostics (Basel) 2019;9:206. 